\title{
Management of pathological femoral fracture secondary to breast cancer in pregnancy: A case report
}

\author{
ANDREA CIAVATTINI $^{1}$, FRANCESCA MANCIOLI ${ }^{1}$, ENRICO PACI $^{2}$ and ROCCO POLITANO ${ }^{3}$ \\ ${ }^{1}$ Women's Health Sciences Department, Polytechnic University of Marche; ${ }^{2}$ Department of Interventional Radiology, \\ Umberto I Hospital; ${ }^{3}$ Department of Orthopedics, Polytechnic University of Marche, Ancona 60100, Italy
}

Received September 17, 2014; Accepted May 1, 2015

DOI: $10.3892 / \mathrm{ol} .2015 .3900$

\begin{abstract}
Bone metastasis resulting from breast cancer in pregnancy is rare. In the literature there are few reports regarding osteolytic lesions in pregnancy and no data on the treatment of such femoral fractures. The present study reports a case of a 29-week primigravida presenting with severe lumbosciatica in the left side, refractory to medical therapy. During neurosurgical examination a spontaneous pathological fracture of the left femur occurred. Damage control orthopedic principals were applied and a biopsy specimen from the femoral lesion was obtained, providing a diagnosis of metastases from breast adenocarcinoma. Cesarean section was performed at 32 gestational weeks. Following delivery, an internal fixator was placed in the left femur for definitive treatment of the fracture and staging of cancer was conducted. Subsequently, adjuvant treatment comprising left mastectomy and percutaneous radiofrequency thermoablation of the sacroiliac lesion were performed. A follow-up one-year following percutaneous radiofrequency thermoablation of the sacroiliac lesion detected no metastatic bone pain, and identified a stable sacroiliac lesion.
\end{abstract}

\section{Introduction}

Breast cancer diagnosed during pregnancy or the postpartum period is known as pregnancy-associated breast cancer (PABC). Breast cancer is the second most common neoplasia in pregnancy, following cervical cancer (1). The incidence of PABC is $~ 1: 3,000-10,000$ in developed countries, an incidence that appears to be increasing as a result of delayed childbearing (2). Breast cancer is most likely to metastasize to bone, followed by the lungs and liver. The overall incidence of bone metastasis from breast advanced neoplasia is $65-75 \%$ (3). The associated complications of bone metastasis include osteolytic lesions, pain, hypercalcemia, fracture and nerve compressions (4). In

Correspondence to: Professor Andrea Ciavattini, Women's Health Sciences Department, Polytechnic University of Marche, 11 Via Corridoni, Ancona 60100, Italy

E-mail: ciavattini.a@libero.it

Key words: breast cancer, pregnancy, osteolytic lesions, radiofrequency thermoablation the literature, there are few reports about osteolytic lesions in pregnancy and no data regarding the treatment of such femoral fractures. Furthermore, to the best of our knowledge, there is currently no information on breast cancer diagnosis secondary to the detection of bone metastases in pregnancy.

The present study reports the management of a case of PABC, which was diagnosed by the presence of bone metastasis, and reviews the relevant literature.

\section{Case report}

In April 2012, a 41-year-old 29-week primigravida was referred to Salesi Hospital (Ancona, Italy) for a severe lumbosciatica in the left side, refractory to medical therapy, and a risk of preterm delivery. The patient's personal and family history was negative for major disease. Spinal magnetic resonance imaging and an electromyography of the left leg were performed, and no abnormalities were detected. Analgesic treatment and neurosurgical counseling were performed. During the neurosurgical exam, a spontaneous pathological fracture of the left femur occurred. Femoral X-rays (Fig. 1) revealed an osteolytic lesion on the distal third of the left femur diaphysis.

Damage control orthopedic principals were applied and an external fixator was placed for temporary femoral stabilization. Initially, the bone lesion was suspected for metastatic breast cancer. A biopsy specimen from the intraosseus lesion was obtained. Bilateral breast ultrasound was conducted and a $6.5 \times 3.5 \mathrm{~cm}$ hypoechoic lesion involving the lower-inner quadrant of the left breast with increased vascularity was identified. Serum analysis of tumor markers [carcinoembryonic antigen, cancer antigen (CA) 19-9, CA 72-4 and CA 15-3] revealed increased levels of CA 72-4. Histological evaluation of the femoral lesion resulted in a diagnosis of metastases from breast adenocarcinoma. Immunohistochemical analysis revealed the presence of estrogen $(40 \%)$ and progesterone receptors (90\%), as well as MIB1 proliferative activity (25\%). Human epidermal growth factor receptor-2/neu expression, evaluated through HercepTest kit (Dako, Glostrup, Denmark), was negative. Neoplastic cells were positive for cytokeratin 7 , mammaglobin and cytokeratin 8/18 (antibody Cam5,2), while negative for cytokeratin 20, thyroid transcription factor 1 and cromogranine. It was suggested by the oncologist that the cancer was staged following delivery, to facilitate planning of the subsequent treatment program. A multidisciplinary meeting 


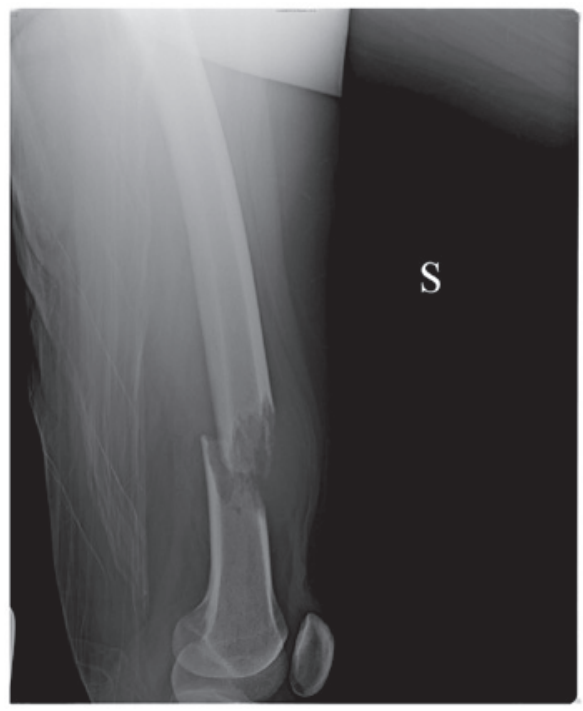

Figure 1. Osteolytic lesion on the distal third of the left femur diaphysis.

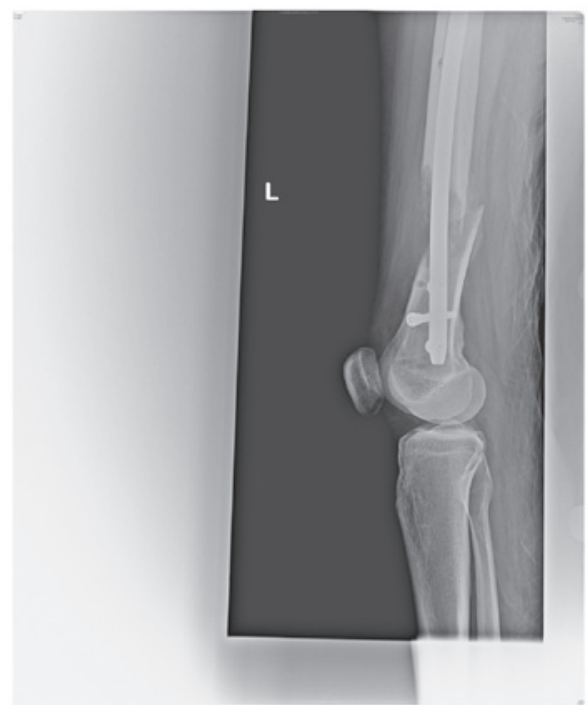

Figure 2. An intramedullary nail was placed for definitive treatment of the femoral fracture following delivery.

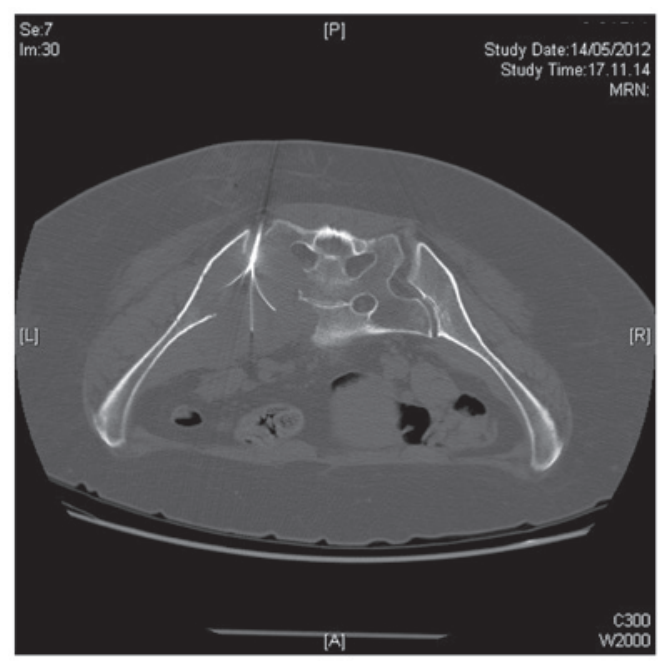

Figure 3. Computed tomography-guided percutaneous radiofrequency thermoablation of the sacroiliac lesion.

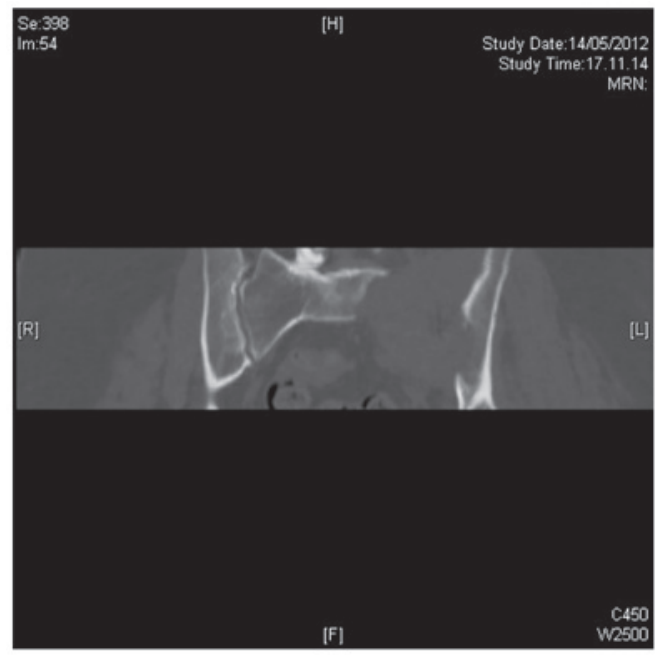

Figure 4. Computed tomography of the sacroiliac lesion.

between the patient, gynecologist, orthopedic surgeon, oncologist and neonatologist was conducted to discuss the appropriate timing of the childbirth. Following receipt of informed consent, a cesarean section was performed at 32 gestational weeks and a vital male was born (weight, $1720 \mathrm{~g}$; Apgar, 8/9).

Following delivery, the external fixation was removed and an intramedullary nail was inserted for definitive treatment of femoral fracture (Fig. 2).

A computed tomography (CT) scan of the chest was performed and identified a left breast lesion and abnormal bilateral intrapulmonary nodules. CT of the neck revealed abnormal laterocervical lymph nodes bilaterally. Cerebral CT was negative, while CT of the abdomen revealed an $8.4 \times 6.2 \mathrm{~cm}$ hyper-vascularized mass on the sacroiliac joint, inducing a wide area of left sacroiliac bone lysis. A bone scintigraphy was performed, and femoral and sacroiliac joint scans demonstrated high uptake.

The patient underwent CT-guided percutaneous radiofrequency thermoablation of the sacroiliac lesion, which resulted in pain relief. The procedure was performed at the Department of Interventional Radiology, Umberto I Hospital (Ancona, Italy) in the CT room under aseptic conditions by an interventional radiologist (Dr Enrico Paci) and an orthopedic surgeon (Dr Rocco Politano) with anesthesiological assistance. The percutaneous approach and needle route were preliminarily assessed by the orthopedic surgeon and interventional radiologist according to imaging data and the position of the lesion with regard to the neurovascular structures. The first CT scan was obtained using an adhesive marker plate with radiopaque coordinates (GuideLines; Beekley Medical, Bristol, CT, USA) to determine the route of the needle from the skin to the lesion. Local anesthesia was administered following skin preparation and sterilization. The Radiofrequency StarBurst $^{\circledR}$, SEMI-FLEX Electrosurgical Device, AngioDynamics (Queensbury, NY, USA) was directly introduced through the soft tissue above the lesion due to erosion of the cortical bone by the mass. The RITA ${ }^{\circledR}$ StarBurst SEMI-FLEX Device has a flexible trocar that is able to bend if required, for example, in order to fit into the CT gantry. The device is able to bend to a radius of $\sim 2$ inches $/ \sim 5 \mathrm{~cm}$. Subsequently, an additional 
CT scan was performed to monitor the needle's direction, and verify the correct deployment of the five electrode/needles. Ablation was performed at two subsequent needle positions, from depth to surface, for a total time of $<15$ min (Fig. 3).

Adjuvant treatment with oral Tamoxifen $(20 \mathrm{mg} / \mathrm{day})$ and intramuscular GnRH-a (Decapeptyl ${ }^{\circledR} ; 11.25$ mg every 3 months) commenced in association with intravenous Zoledronic acid (4 mg every 3-4 weeks), prescribed due to its action on osteoclasts and antitumor properties. Follow-up CT of the chest and abdomen was performed two months subsequent to thermoablation of the sacroiliac lesion and the commencement of adjuvant treatment. The chest $\mathrm{CT}$ revealed a reduction in the size of the left breast lesion $(4 \times 5 \mathrm{~cm})$, and abdominal CT revealed a significant decrease in the sacroiliac lesion with a maximum diameter of $6 \mathrm{~cm}$. Four months following delivery, the patient underwent monolateral mastectomy.

A follow-up 18 months post-percutaneous radiofrequency thermoablation of the sacroiliac lesion, the patient exhibited no metastatic bone pain and CT revealed a reduction in the lesion and bone formation of anterior cortical bone of the sacroiliac. Furthermore, femoral X-ray indicated fracture healing and a reduction of the lesion. $\mathrm{CT}$ of the chest and abdomen was also conducted and revealed analogous findings compared with previous CT scans (Fig. 4). Written informed consent was obtained from the patient for the publication of this study.

\section{Discussion}

Though breast cancer is the second most common cancer in pregnant women, it occurs relatively infrequently (2), and metastatic breast cancer during pregnancy is a rare event. In the literature there are few studies regarding this topic. A review of the literature identified eleven reported cases of metastatic breast cancer in pregnant women. The primary diagnosis of breast cancer had been reached prior to pregnancy in nine of these cases (5). These nine women subsequently presented with metastasis or breast cancer progression during pregnancy. Initial diagnosis of metastatic disease in pregnancy was made in the remaining two cases $(6,7)$. It is well established that bone is a frequent site for metastases among patients with breast cancer, with a reported frequency of $65-75 \%$ (3). The present case is a notable example of breast cancer initially diagnosed in pregnancy at 30 gestational weeks, as a result of a pathological femoral fracture. It is well known that pregnancy induces reversible bone turn-over and loss, particularly in the second and third trimester, with a significant decrease in bone mineral density (8). It may be hypothesized that these changes in bone metabolism enhance the severity of bone metastasis in pregnancy, considering that the bone microenvironment has a crucial role in the development of malignant bone lesions. In fact, an increase in bone resorption results in enhanced release of growth factors, which may promote bone tumor formation (9).

Furthermore, since there are few studies regarding osteolytic lesions in pregnancy and no data on the treatment of such femoral fractures in the literature, to the best of our knowledge, the present case report is the first to report the management of pathological femoral fracture secondary to breast cancer in pregnancy. Traumatic injuries are detected in $6-7 \%$ of pregnant women (10). These are the most common non-obstetric risk factors for miscarriage and preterm delivery. Damage control orthopedic principles are applied when early total care is impossible (11). In addition, previous studies have reported rare cases of fractures due to transient osteoporosis during pregnancy, which may be treated with internal fixation or arthroplasty $(10,12)$.

A significant dilemma for obstetricians and oncologists arises following the diagnosis of metastasis from breast cancer in pregnancy. This is due to the fact that metastatic cancer must be treated as incurable and the quality of life of the patient, as well as the newborn, must be considered. In the present case, percutaneous radiofrequency thermoablation of the sacroiliac lesion had an essential role in improving the quality of life of the patient by exerting marked pain relief.

\section{References}

1. Fedarapalli P and Jain S: Breast cancer in pregnancy. J Obstet Gynaecol 26: 1-4, 2006.

2. Genin AS, Lesieur B, Gligorov J, Antoine M, Selleret L and Rouzier R: Pregnancy-associated breast cancers: Do they differ from other breast cancers in young women? Breast 21: 550-555, 2012.

3. Coleman RE: The clinical use of bone resorption markers in patients with malignant bone disease. Cancer 94: 2521-2533, 2002.

4. Mundy GR: Metastasis to bone: Causes, consequences and therapeutic opportunities. Nat Rev Cancer 2: 584-593, 2002.

5. Litton JK, Theriault RL and Gonzalez-Angulo AM: Breast cancer diagnosis during pregnancy. Womens Health (Lond Engl) 5: 243-249, 2009.

6. El-Safadi S, Wuesten O and Muenstedt K: Primary diagnosis of metastatic breast cancer in the third trimester of pregnancy: A case report and review of the literature. J Obstet Gynaecol Res 38: 589-592, 2012.

7. Azim HA Jr and Peccatori FA: Treatment of metastatic breast cancer during pregnancy: We need to talk! Breast 17: 426-428, 2008.

8. Tranquilli AL, Giannubilo SR and Corradetti A: Ultrasound measurement of pregnancy-induced changes in maternal bone mass: A longitudinal, cross-sectional and biochemical study. Gynecol Endocrinol 18: 258-262, 2004.

9. Gnant M, Dubsky P and Hadji P: Bisphosphonates: prevention of bone metastases in breast cancer. Recent Results Cancer Res 192: 65-91, 2012.

10. Ahmadi A, Fakheri T, Amini-Saman J, Amanollahi O, Mordi M, Nasrabadi MA, Gholipour Y, Dehghani R and Bazargan-Hejazi S: Traumatic injuries in pregnant women: A case of motor vehicle accident for 'Ground Round' discussion. J Inj Violence Res 3: 55-59, 2011.

11. Barraco RD, Chiu WC, Clancy TV, Como JJ, Ebert JB, Hess LW, Hoff WS, Holevar MR, Quirk JG, Simon BJ and Weiss PM; EAST Practice Management Guidelines Work Group: Practice management guidelines for the diagnosis and management of injury in the pregnant patient: The EAST Practice Management Guidelines Work Group. J Trauma 69: 211-214, 2010.

12. Emami MJ, Abdollahpour HR, Kazemi AR and Vosoughi AR: Bilateral subcapital femoral neck fractures secondary to transient osteoporosis during pregnancy: A case report. J Orthop Surg (Hong Kong) 20: 260-262, 2012. 